\title{
Investigation of high temperature superconductivity through microwave absorption method
}

\author{
C M SRIVASTAVA $^{*}, \mathrm{~N}_{\text {VENKATRAMANI }}^{+}$, A DATTA $^{*}$ \\ and $\mathrm{N} \mathrm{S} \mathrm{H} \mathrm{RAO}^{+}$ \\ ${ }^{*}$ Department of Physics and ${ }^{+}$Advanced Centre for Research in Electronics. Indian Institute \\ of Technology, Bombay 400076 . India
}

\begin{abstract}
The field dependence and near zero magnetic field microwave absorption as a function of of power in $\mathrm{YBa}_{2} \mathrm{Cu}_{3} \mathrm{O}_{7-\delta}$ and $\mathrm{Bi}-\mathrm{Ca}-\mathrm{Sr}-\mathrm{Cu}-\mathrm{O}$ has been studied using a varian ESR spectrometer. A model of microwave absorption built on diamagnetic tensor susceptibility has been proposed which explains the observed results satisfactorily.
\end{abstract}

Keywords. Y-Ba-Cu-O; Bi-Ca-Sr-Cu-O; microwave absorption; ESR.

\section{Introduction}

The microwave absorption in oxidic superconductors has been extensively studied (Durny et al 1987; Blazey et al 1987; Stankowski et al 1987; Khachaturyan et al 1987; Sastry et al 1988; Shvachko et al 1989; Pozek et al 1989). In a dc magnetic field microwave absorption shows a maximum near zero field and begins to decrease as the field is increased. At some fields depending on the composition and microstructure the absorptions tends to saturate (Shvachko et al 1989). Despite considerable effort the origin of absorption is not understood. To investigate the nature of the absorption we have investigated the microwave absorption properties of superconducting ceramics of the composition $\mathrm{YBa}_{2} \mathrm{Cu}_{3} \mathrm{O}_{7-\delta}$ and $\mathrm{Bi}_{2} \mathrm{Ca}_{3} \mathrm{Sr}_{2} \mathrm{Cu}_{2} \mathrm{O}_{8 \pm y}$.

\section{Experimental}

The ceramic samples were prepared by the standard solid-state reaction procedure using powder of $\mathrm{Y}_{2} \mathrm{O}_{3}, \mathrm{BaCO}_{3}, \mathrm{CuO}, \mathrm{Bi}_{2} \mathrm{O}_{3}, \mathrm{SrCO}_{3}$ and $\mathrm{CaCO}_{3}$. The X-ray diffraction pattern and the temperature dependence of resistance show that the $\mathrm{YBa}_{2} \mathrm{Cu}_{3} \mathrm{O}_{7-\delta}$ systems are single-phase materials with $T_{c}=90 \mathrm{~K}$ whereas $\mathrm{Bi}_{2} \mathrm{Ca}_{3} \mathrm{Sr}_{2} \mathrm{Cu}_{2} \mathrm{O}_{8+y}$ system is a mixed phase system with zero resistance at $80 \mathrm{~K}$. All ESR measurements were carried out on a variable temperature standard E-varian $9.2 \mathrm{GHz}$ ESR spectrometer. Spherical samples with diameters $1 \mathrm{~mm}$ to $2.5 \mathrm{~mm}$ and powdered sampie $(d \sim 10 \mu \mathrm{m})$ were used for the measurements. A rectangular $\mathrm{TE}_{102}$ cavity with the microwave power varying from $0.005 \mathrm{~mW}$ to $15 \mathrm{~mW}$ was used. The external magnetic field, oriented perpendicular to the microwave field $\left(h_{\mathrm{rf}}\right)$, was varied from 0 to $6.5 \mathrm{KOe}$. Hfmodulation $(100 \mathrm{kHz})$ had an amplitude in the range $0.5 \mathrm{Oe}$ to $2 \mathrm{Oe}$.

\section{Results}

The field dependence of the microwave power loss $(\mathrm{d} P / \mathrm{d} H)$ with the magnetic field for different superconductors is presented in figures 1 and 2 . The absence of any ESR signal 
at room temperature for $\mathrm{YBa}_{2} \mathrm{Cu}_{3} \mathrm{O}_{7-\delta}$ samples taken from two different batches as denoted by $\mathrm{R} 1$ and $\mathrm{R} 1 \mathrm{~A}$ indicates that the samples are of a good quality. Figure 1 shows the low-field microwave absorption at $77 \mathrm{~K}$ for different samples with field span of 0 to 400 Oe. Figure 2 shows the hysteresis behaviour of a polycrystalline sphere and the powder sample taken from the same batch $\mathrm{R} 1$ with field span of 0 to $6500 \mathrm{Oe}$. The dependence of microwave power absorption on the $\mathrm{rf}$-power near zero magnetic field is presented in figure 3. The magnetization curves for the spherical and powdered samples obtained at $77 \mathrm{~K}$ by a Faraday balance are given in figure 4 . It is noted that the hysteresis behaviour of the absorption and the magnetization curve is similar.

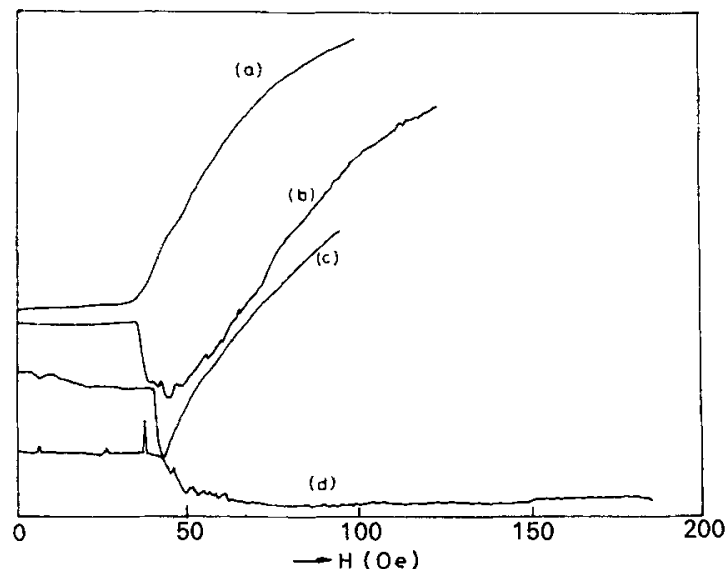

Figure 1. Low field microwave absorption at $77 \mathrm{~K}$ with field span of 0 to 400 Oe. (a): 1-2-3 sphere from batch RIA, (b): 1-2-3 sphere from batch R1, (c): BSCCO sphere and (d) 1-2-3 powder from batch $R 1$.

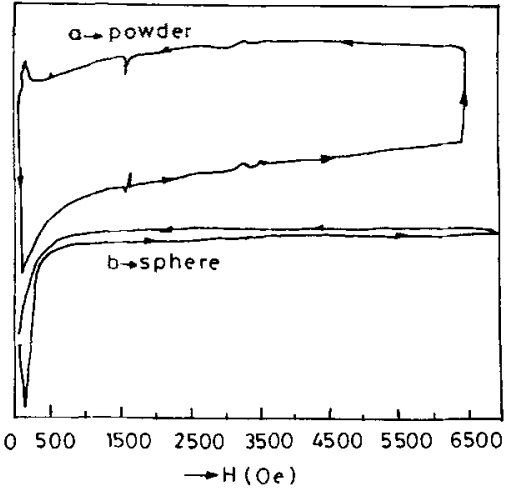

Figure 2. Hysteresis behaviour of a polycrystalline sphere and the powder sample taken from the same batch $\mathrm{R} 1$ with field span of 0 to $6500 \mathrm{Oe}$. (a) powder, and (b) sphere

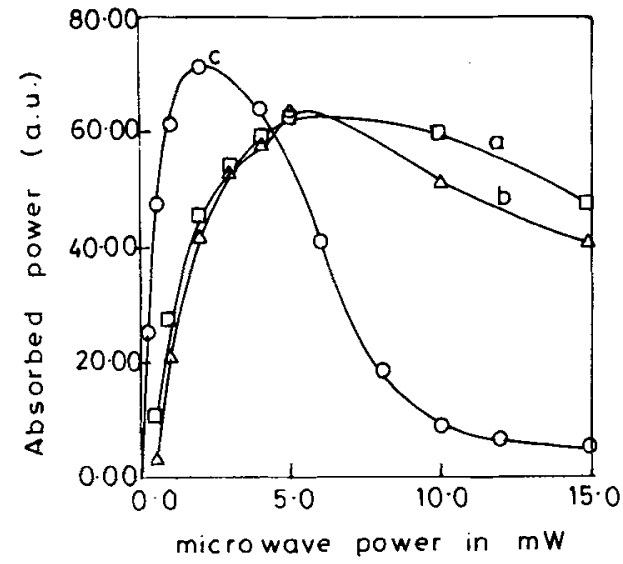

Figure 3. Dependence of microwave power absorption on the rf-power near zero magnetic field at $77 \mathrm{~K}$. (a) 1-2-3 sphere from batch R1, (b) 1-2-3 sphere from batch R1A, and (c) BSCCO sphere.

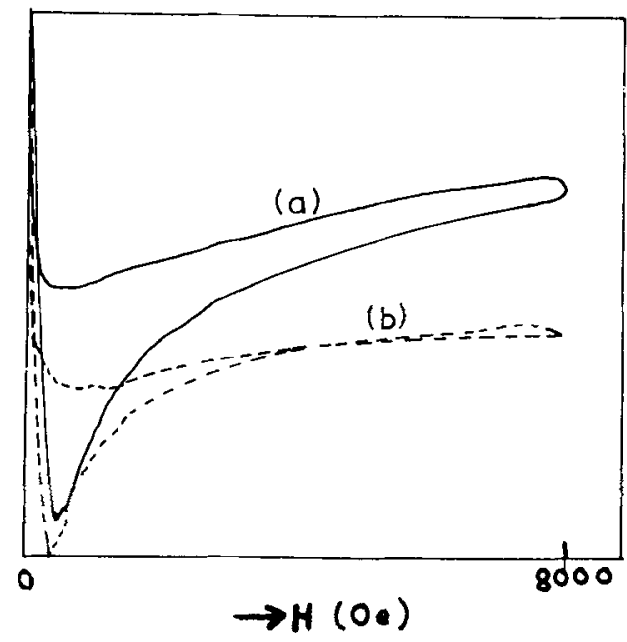

Figure 4. Magnetization curve for (a) powder and (b) spherical samples obtained at $77 \mathrm{~K}$ with a field span of 0 to $8000 \mathrm{Oe}$. 


\section{Discussion}

Several mechanisms for the large microwave absorption below $T_{c}$ have been proposed. Amongst these are absorption due to diamagnetic a.c. susceptibility of a granular superconductor (Durny et al 1987). Suggestions that these may arise from changes in $x_{\text {dia }}$ upon transition from the Meissner phase to the mixed phase have also been made (Blazey et al 1988; Rettori et al 1987). Attempts have also been made to attribute it to flux penetration through weak links between superconducting regions (Stankowski et al 1987; Khachaturyan et al 1987; Shrivastava 1987).

Based on the present study and the data available in literature the significant experimental results are as follows. (i) The zero field microwave absorption intensity follows approximately the $|\overrightarrow{\mathbf{M}}|$ vs $H$ curve where $\overrightarrow{\mathbf{M}}$ is the magnetization of the sample (ii) Initially the absorption is independent of the biasing field, $H_{0}$. However at a field $H_{0}^{*}$ there is a discontinuity in the intensity of absorption and further increase in $\mathrm{H}_{0}$ leads to a rapid decrease in absorption leading to saturation at a field of the order of $200 \mathrm{Oe}$ for polycrystalline spheres. For powder samples saturation is not reached even at $6500 \mathrm{Oe}$.

(iii) There is hysteresis in the absorption curve which is very pronounced in powder samples.

We propose a model based on the interaction between the Meissner and shielding currents produced by the bias, modulation and $\mathrm{ff}$ fields. Since the rf field is orthogonal to the bias and modulation fields the relation between magnetization and the field could be described by a complex tensor susceptibility $\mathbf{X}$.

We obtain for

$$
\begin{aligned}
& H_{r f}=H_{x 1} \exp (j \omega t) \\
& H_{\text {bias }}=H_{y 0}+H_{y 1} \exp (i \Omega t)
\end{aligned}
$$

and $H<H^{*}$ the power loss from Meissner currents

$$
\left.P_{M}=a \lambda^{2}(\omega)^{2}+\Omega^{2}\right)^{1 / 2} x_{x y}^{\prime \prime} H_{x 1} H_{y 1}+a^{2} \lambda\left(\omega x_{x x}^{\prime \prime} H_{x 1}^{2}+\Omega x_{y y}^{\prime \prime} H_{y 1}^{2}\right) .
$$

Here $\lambda$ is the penetration depth and $a$ is the radius of the spherical sample. For $H>H^{*}$ the superconductor is in the mixed state. The power absorbed due to shielding current is given by

$$
P_{s}=a^{3} \Omega x_{y y}^{\prime \prime} H_{y 0} H_{y 1} .
$$

The observed microwave absorption in the ESR cavity is given by $\mathrm{d}\left(P_{M}+P_{S}\right) / \mathrm{d} H_{y 1}$. For $H<H^{*}$ contribution comes from $P_{M}$ while for $H>H^{*}, P_{S}$ contributes. We thus have

$$
\begin{aligned}
& \partial P_{M} / \partial H_{y 1}=a \lambda^{2}\left(\omega^{2}+\Omega^{2}\right)^{1 / 2} x_{x y}^{\prime \prime} H_{x 1}+2 a^{2} \lambda \Omega x_{y y}^{\prime \prime} H_{y 1} \quad\left(H<H^{*}\right) \\
& \mathrm{d} P_{s} / \mathrm{d} H_{y 1}=a^{3} \Omega x_{y y}^{\prime \prime} H_{y 0} \sim a^{3} \Omega x_{y y}^{\prime \prime} x^{\prime-1} M_{y 0} \quad\left(H<H^{*}\right) .
\end{aligned}
$$

If $x^{\prime}$ in (5) is independent of $H$ the microwave loss in the region $H>H^{*}$ is proportional to magnetization as observed. From (4) we observe that in the region $H<H^{*}$, absorption is independent of $H_{y c}$. It is also proportional to the rf and modulation field amplitude as observed. The peak in absorption seen at $H_{x 1}=0.12 \mathrm{Oe}$ (calculated by using the $Q$-value of the cavity) for 1-2-3 sample is due to the onset of shielding current which suppress $P_{M}$. 
In conclusion we can say that the present model of microwave absorption built on diamagnetic tensor susceptibility explains the observed data satisfactorily.

\section{References}

Blazey K W, Muller K A, Bednorz J G, Berlinger W, Amoretti G, Bewrluggium E, Vera A and Mattacotta F C 1987 Phys. Rev. B36 7241

Blazey K W, Portis A M and Bednorz J G 1988 Solid State Commun. 651153

Durny R, Hautala J, Ducharme S, Lee B, Symko G, Taylor P C, Zheng D and Xu J A 1987 Phys. Rev. B36 2361

Khachaturyan K, Weber E R, Tejedor P, Stacy A M and Portis A M 1987 Phys, Rev. B36 8309

Pozec M, Dulcic A and Rakvin B 1989 Solid State Commun. 70889

Rettori C, Davidov D, Belaish I and Felner I 1987 Phys. Rev. B36 4028

Sastry M D, Kadam R M, Babu Y, Dalvi A G I, Gopalakrishnan I K, Sastry P V P S S and Iyer R M 1988 Physica C153-155 1667

Shrivastava K N 1987 J. Phys, C20 1789

Shvachko Yu N, Khusainov D Z, Ramanyukha A A and Ustinov V V 1989 Solid State Commun. 69611

Stankowski J, Kohol P K, Dalal N S and Moodera J S 1987 Phys. Rev. B36 7126 